\title{
Discriminating between the UV-A, UV-B and UV-C regions by novel \\ Biologically Inspired Photochromic Fuzzy Logic (BIPFUL) Systems; a Detailed Comparative Study
}

\author{
Pier Luigi Gentili, ${ }^{*^{a}}$ Amanda L. Rightler, ${ }^{\mathrm{a}, \mathrm{b}}$ B. Mark Heron, ${ }^{\mathrm{c}}$ Christopher D. Gabbutt ${ }^{\mathrm{c}}$

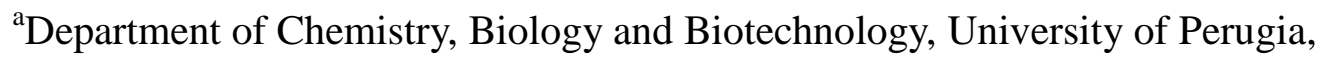 \\ Via Elce di sotto 8, 06123 Perugia, Italy \\ ${ }^{\mathrm{b}}$ Department of Chemistry and Biochemistry, University of Tulsa, Tulsa, Oklahoma, USA \\ ${ }^{\mathrm{c}}$ Department of Chemical and Biological Sciences, School of Applied Science, \\ University of Huddersfield, Queensgate, Huddersfield, HD1 3DH, UK
}

\begin{abstract}
This work presents the analysis of the deterministic part of human colour perception at the computational, algorithmic and implementation levels. At the computational and algorithmic levels, we invoke the theory of Fuzzy sets. At the implementation level, we present Biologically Inspired Photochromic Fuzzy Logic (BIPFUL) systems that are useful to discriminate between the three regions of the UV spectrum (UV-A, UV-B and UV-C). Such BIPFUL systems have been devised by imitation of the human photosensitive system on retina. After transforming the absorption spectra, originated by the BIPFUL systems under UV-A, UV-B and UV-C, in chromaticity coordinates and vectors in CIE L*a*b* spaces, we have compared their performances through the determination of two parameters: The Colourability and the Eucledian distance. Finally, the guiding principles for the design of new BIPFUL systems are outlined.
\end{abstract}




\section{Keywords}

Fuzzy sets; Spirooxazines; Naphthopyrans; Colours; Colourability; Systems Chemistry; Artificial Intelligence.

\section{Introduction}

Nowadays, science is spurred to win the Complexity challenges. There are two types of these challenges. One type regards Natural Complexity and the other type regards Computational Complexity. As far as the Natural Complexity is concerned, the challenges are understanding and predicting the behaviour of complex systems like the planet earth and its climate, ecosystems, living beings, the human brain, social organizations and the macro-economy. As far as the Computational Complexity is concerned [1], the challenges are solving accurately and in reasonable time the NonDeterministic Polynomial (NP) problems having large dimensions. Examples of NP problems are the well-known "Salesman problem" or the determination of the energy of molecules that is a compelling problem for chemists. Another challenge in the field of Computational Complexity is that of finding universally valid and effective algorithms for recognizing variable patterns like human faces and voices, handwritten cursive words or numbers, fingerprints, patterns in medical diagnosis, patterns in apparently uncorrelated variables, and distinguishing stochastic from chaotic aperiodic dynamics.

How can we tackle the Complexity Challenges? Since Complexity means handling a huge amount of data, we need to increase our computational rate and establish new ways to process data. One strategy consists of developing the interdisciplinary research line of Natural Computing [2]. Researchers, working in the field of Natural Computing, draw inspiration from nature to propose 
new algorithms, new materials to compute and new models to interpret Complexity, based on the rationale that every natural transformation is a kind of computation.

A prodigious source of inspiration in nature is the human nervous system (HNS) that has human intelligence as its own emergent property. The HNS is a complex system of billions of nerve cells that allows a human to handle both accurate and vague information by computing with numbers and words; it facilitates decision formation in complex situations and readily recognizes variable patterns. Therefore, it is worthwhile trying to deeply understand the working principles of the HNS and human intelligence in order to reproduce them artificially. In our group, we are trying to implement some basic function of human intelligence at the chemical level. In other words, we are trying to develop a Chemical Artificial Intelligence [3]. To succeed in our project, we are following the methodology, proposed by the cognitive scientists Gallistel and King [4] and by the neuroscientist Marr [5], that is effective in dealing with any complex system. It consists in analyzing a complex system, like the HNS, at three levels. First, at the computational level; then, at the algorithmic level, and finally, at the implementation level. The analysis at the computational level requires identification of the inputs, the outputs and the computations that the system performs. The analysis at the algorithmic level consists in formulating algorithms that might carry out those computations. Finally, the analysis at the implementation level consists in finding out mechanisms and artificial systems that might implement the formulated algorithms.

In this work, we first, analyze the human power of discriminating colours at the computational and algorithmic levels by invoking the theory of fuzzy sets. Then, we implement the formulated algorithm by using chemical systems consisting of either three or four or five photochromic compounds among those depicted in Fig. 1. Such systems, which we have termed Biologically Inspired Photochromic Fuzzy Logic (BIPFUL) systems, have been determined to extend the human ability to discriminate frequencies of the electromagnetic spectrum from the visible into the UV region. 
<smiles>CN1c2ccccc2C(C)(C)C12C=Nc1c(ccc3cc([N+](=O)[O-])ccc13)O2</smiles>

(1)<smiles>C1=CC(c2ccccc2)(c2ccc(N3CCOCC3)cc2)Oc2cc(N3CCOCC3)c3ccccc3c21</smiles><smiles>COc1ccc(C2(c3ccc(OC)cc3)C=Cc3c(C)c(C)c4ccccc4c3O2)cc1</smiles><smiles>C1=CC(c2ccccc2)(c2ccccc2)Oc2cc(N3CCOCC3)c3ccccc3c21</smiles>

(4)<smiles>Fc1ccccc1C1(c2ccc(N3CCCC3)cc2)C=Cc2c(ccc3ccccc23)O1</smiles>

(5)

Fig.1 Structures of the five photochromic compounds used in this study: (1) is 1,3-dihydro1,3,3-trimethyl-8'-nitro-spiro[2H-indole-2,3'-[3H]naphth[2,1-b][1,4]oxazine]; (2) is 2,2-bis(4methoxyphenyl)-5,6-dimethyl-2H-naphtho[1,2-b]pyran;

(3) is 6-morpholino-3-(4morpholinophenyl)-3-phenyl-3H-naphtho[2,1-b]pyran; (4) is 3,3-diphenyl-6-morpholino-3Hnaphtho[2,1-b]pyran; (5) is 3-(2-fluorophenyl)-3-(4-pyrrolidinophenyl)-3H-naphtho[2,1-b]pyran.

Finally, we identify the best BIPFUL systems for discriminating between the three main regions of the ultraviolet spectrum, i.e. UV-A, UV-B and UV-C, by transforming their absorption spectra in colour coordinates and determining the Colourability and the Eucledian distance between pairs of vectors in the CIE L*a*b* space.

This work shows how systems of properly chosen photochromic compounds have the computing power of discriminating UV frequencies. It demonstrates that Systems Chemistry [6] may be really useful for the development of Chemical Artificial Intelligence [3]. 


\section{Experimental}

\subsection{Materials and facilities}

Compound (1) 1,3-dihydro-1,3,3-trimethyl-8'-nitro-spiro[2H-indole-2,3'-[3H]naphth[2,1b][1,4]oxazine] was supplied by Great Lakes Chemical Italia S.r.l. Compounds (2) 2,2-bis(4methoxyphenyl)-5,6-dimethyl-2H-naphtho[1,2-b]pyran, (3) 6-morpholino-3-(4-morpholinophenyl)3-phenyl-3H-naphtho[2,1-b]pyran, (4) 3,3-diphenyl-6-morpholino-3H-naphtho[2,1-b]pyran and (5) 3-(2-fluorophenyl)-3-(4-pyrrolidinophenyl)-3H-naphtho[2,1-b]pyran have been synthesized according to the procedures described in reference [7].

The spectrophotometric measurements were carried out by using a Hewlett-Packard 8453 diode array spectrophotometer. The photochromic species were dissolved in acetonitrile (from Fluka, $\geq 99.8 \%$ ) in concentrations ranging from $10^{-5}$ to $10^{-3} \mathrm{~mol} \mathrm{dm}^{-3}$, without experiencing any solubility limit.

A $125 \mathrm{~W}$ Xe lamp, filtered by a Jobin-Yvon H10 UV monochromator and focused on the sample through a $0.6 \mathrm{~cm}$ diameter silica optical fiber, was used as the irradiation source. A mercury lamp (Mineralight Compact 4-Watt UV lamp) was used to irradiate at $254 \mathrm{~nm}$. The irradiation intensity was determined by potassium ferrioxalate actinometry and by performing spectroradiometric measurements. For the latter measurements, we used an irradiance-calibrated AvaSpec2048-2 spectrometer (Avantes, NL) provided with a 200 micrometer diameter optical fiber (FCUVIR200-2ME, Avantes) and an $8 \mathrm{~mm}$ active area cosine corrector (CC-UV-VIS/NIR, Avantes). The spectroradiometer operated in the $171-1100 \mathrm{~nm}$ range (300 lines per mm grating) and was equipped with an AvaBench-75 optical bench, a 25 micrometer slit which produced $1.2 \mathrm{~nm}$ FWHM (Full Width at Half Maximum) spectral resolution and a 2048 pixel CCD detector.

The profiles of UV-A, UV-B and UV-C radiations used to study the response of the BIPFUL systems are depicted in Fig. 2, below. The UV-A and UV-B are the output of the $125 \mathrm{~W}$ Xe lamp, 
whereas UV-C is the output of the Mineralight Compact 4-Watt UV lamp. Regarding the UV-C, we considered only radiation having wavelength longer than $250 \mathrm{~nm}$.

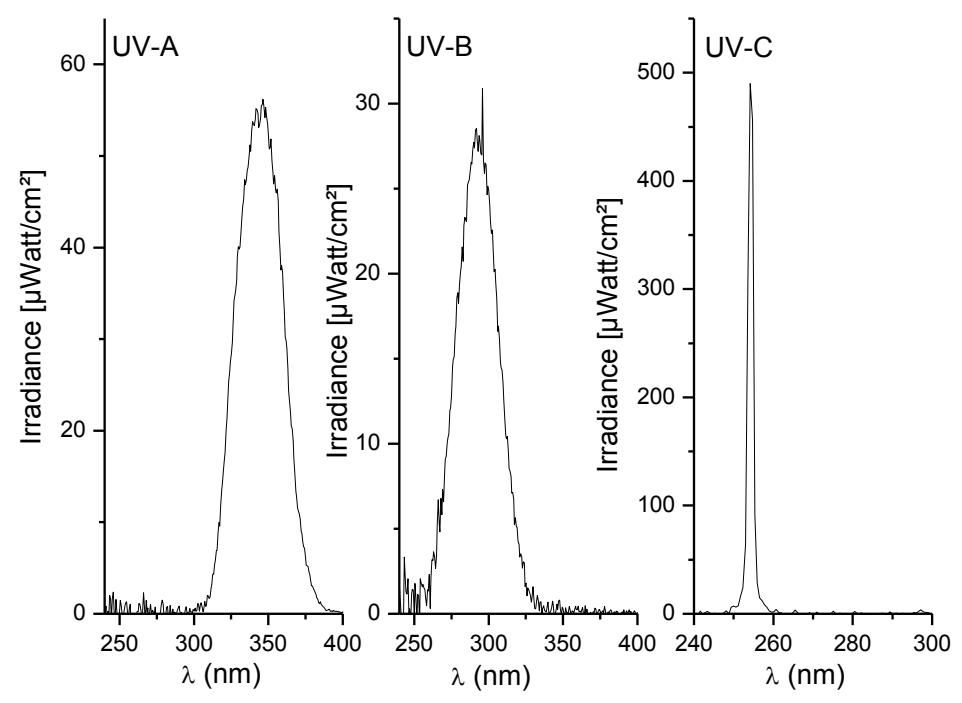

Fig. 2 Spectral profiles of the UV-A, UV-B and UV-C radiation used to study the responses of the BIPFUL systems.

\subsection{Computational methods}

In order to transform the transmittance spectra, recorded at the photostationary states of compounds (1) - (5), in the CIE XYZ tristimulus values, the following integrals were used:

$$
\begin{aligned}
& X=\frac{1}{k} \int_{360}^{800} D(\lambda) T(\lambda) \bar{x}(\lambda) d \lambda \\
& Y=\frac{1}{k} \int_{360}^{800} D(\lambda) T(\lambda) \bar{y}(\lambda) d \lambda \\
& Z=\frac{1}{k} \int_{360}^{800} D(\lambda) T(\lambda) \bar{z}(\lambda) d \lambda
\end{aligned}
$$

In equation (1), $\bar{x}, \bar{y}, \bar{z}$ are the colour-matching functions whereby the CIE (Commision Internationale de l'Éclairage) standardized the sensitivity of human eye in 1964; $D(\lambda)$ is the energy 
distribution of the CIE normalized illuminant D65 (which closely matches that of normal daylight); $\mathrm{T}(\lambda)$ is the transmittance spectrum, and $k$ is a normalization factor defined in such a way that a sample with a uniform transmittance $T(\lambda)=1$ for $\lambda \in[360-800]$ gives a luminance component $\mathrm{Y}=$ 1:

$$
k=\int_{360}^{800} D(\lambda) \bar{y}(\lambda) d \lambda
$$

The XYZ tristimulus values were transformed into the CIE L*a*b* coordinates by the algorithms (3), (4) and (5), which are based on the tristimulus values determined for the reference white (i.e., $\mathrm{X}_{\mathrm{W}}=0.94811, \mathrm{Y}_{\mathrm{W}}=1, \mathrm{Z}_{\mathrm{W}}=1.0730$ ):

$$
\begin{array}{ll}
L^{*}=116\left(\frac{Y}{Y_{W}}\right)^{1 / 3}-16 \quad \text { for }\left(\mathrm{Y} / \mathrm{Y}_{\mathrm{W}}\right)>0.008856 \\
L^{*}=903.3\left(Y / Y_{W}\right) \quad \text { otherwise } \\
a^{*}=500\left(f\left(\frac{X}{X_{W}}\right)-f\left(\frac{Y}{Y_{W}}\right)\right) \\
b^{*}=200\left(f\left(\frac{Y}{Y_{W}}\right)-f\left(\frac{Z}{Z_{W}}\right)\right)
\end{array}
$$

where $f(t)=t^{1 / 3}$ for $t>0.008856$, whereas $f(t)=7.787 t+16 / 116$ otherwise.

The chromaticity coordinates $x, y$ and $z$ were determined through equation (6):

$$
\begin{aligned}
& x=X /(X+Y+Z) \\
& y=Y /(X+Y+Z) \\
& z=Z /(X+Y+Z)
\end{aligned}
$$

From equation (6), it derives that $x+y+z=1$. The values of the chromaticity coordinates were used to estimate the Colourability formulated from the definition of Shannon's Information, according to equation (7): 
$C=I_{C o l}-I_{U n}=x_{C o l} \log _{2}\left(x_{C o l}\right)-x_{U n} \log _{2}\left(x_{U n}\right)+y_{C o l} \log _{2}\left(y_{C o l}\right)-y_{U n} \log _{2}\left(y_{U n}\right)+z_{C o l} \log _{2}\left(z_{C o l}\right)-z_{U n} \log _{2}\left(z_{U n}\right)$

wherein $x_{U n}, y_{U n}, z_{U n}$ and $x_{C o l}, y_{C o l}, z_{C o l}$ are the values of the chromaticity coordinates for the uncoloured (Un) and coloured forms (Col), respectively (see reference [8] and the text below, for more information). Since the chromaticity coordinates of the solutions before irradiation assumed the values of a white hue (for example, $\mathrm{x}_{\mathrm{Un}}=0.3187, \mathrm{y}_{\mathrm{Un}}=0.3403, \mathrm{z}_{\mathrm{Un}}=0.3410$ ), when the solutions under irradiation gave rise to grey hues, the resulting $\mathrm{C}$ was low.

\section{Results and discussion}

\subsection{Computational and Algorithmic analysis of the deterministic part of human colour perception.}

The human sensory system consists of seven specialized elements that are the auditory, the visual, the olfactory, the gustatory, the somatosensory, the thermoreceptor, and the nociceptor systems [9]. Each of these elements has a hierarchical structure. At the smallest level, there is a collection of distinct molecular switches. At an upper level, we have a set of distinct sensory cells. Usually, a sensory cell contains many replicas of one particular molecular switch. Finally, at the highest level, we have many replicas of the different types of sensory cells spread on a sensory tissue within a particular organ. The multiple information of a stimulus, that is, its modality (M), intensity $\left(\mathrm{I}_{\mathrm{M}}\right)$, spatial distribution $\left(\mathrm{I}_{\mathrm{M}}(\mathrm{x}, \mathrm{y}, \mathrm{z})\right)$ and time evolution $\left(\mathrm{I}_{\mathrm{M}}(\mathrm{t}, \mathrm{x}, \mathrm{y}, \mathrm{z})\right)$, is encoded hierarchically [10]. The mechanism of encoding stimuli information can be rationalized by invoking the theory of fuzzy sets [10]. A fuzzy set [11] is different from a classical set, because it breaks the law of excluded middle. In fact, an item may belong to a fuzzy set and its complement at the same time, with equal or different degree of membership. The degree of membership $(\mu)$ of an element to a fuzzy set can be any real number included between 0 and 1 . 
Focusing on the human visual system [12], we find that it is founded upon four photoreceptor proteins: each one has its own absorption spectrum in the visible region of the electromagnetic spectrum. The four photosensitive proteins have the 11-cis retinal as their own chromophore. The differences in their spectra stem from the amino-acid composition of the pockets embedding the chromophore. The first step of vision is the photo-isomerization of the photochromic 11-cis retinal to all-trans retinal. The absorption spectra of the four photoreceptor proteins behave as "molecular fuzzy sets". The modality of a light stimulus, that is its spectral composition, is encoded as degrees of membership of the light to the "molecular fuzzy sets", i.e. as fuzzy information at the molecular level $\left(\bar{\mu}_{M L}\right)$. At an upper level, the visual system consists of four types of photosensitive cells: one rod and three types of cones. Each one of these four types of cells has millions of replicas of one particular photoreceptor protein. The photosensitive cells play like "cellular fuzzy sets". The information regarding the intensity of the stimulus at the different frequencies is encoded as degrees of membership of the stimulus to the "cellular fuzzy sets". In other words, it is encoded as fuzzy information at the cellular level $\left(\bar{\mu}_{C L}\right)$. Finally, we have millions of replicas of the four types of photosensitive cells covering the retina. The cones are more concentrated on the center of the retina, called fovea, whereas the rods are more abundant on the periphery of the retina. The information regarding the spatial distribution of the light stimulus is encoded as degrees of membership of the stimulus to the array of photosensitive cells, i.e. it is encoded as fuzzy information at the tissue level $\left(\bar{\mu}_{T L}\right)$. Finally, the total information of the light stimulus will be a matrix of data whose shape reproduces the array of rods and cones on the retina. Each element of the matrix will be the product of two contributions, $\bar{\mu}_{M L} * \bar{\mu}_{C L}$, as expressed in equation (8), below:

$$
\bar{\mu}_{M L} * \bar{\mu}_{C L}=\Phi_{P C} I_{0, \lambda}\left(1-10^{-\varepsilon_{i} C_{i} l}\right)
$$

\footnotetext{
${ }^{1}$ The product $\bar{\mu}_{M L} * \bar{\mu}_{C L}$ is included between 0 and 1 if at the denominator of its definition we add the term ( $\mathrm{n}_{\text {MAX } c \rightarrow \mathrm{t}}$ )/ $\Delta \mathrm{t}$ that is the maximum number of photoisomerisation 11-cis- $\rightarrow$ all-trans that can occur, per unit time, within a cell.
} 
wherein $\Phi_{P C}$ is the quantum yield of the photo-isomerization of 11-cis retinal, $I_{0, \lambda}$ is the intensity of the stimulus at wavelength $\lambda, \varepsilon_{i}$ is the absorption coefficient of the $i$-th photoreceptor protein, $C_{i}$ is its concentration within a cell and $l$ is the length of the cell that represents the optical path length. Light stimuli having different colours are transduced in matrices with different values of their elements.

\subsection{Implementation of Biologically Inspired Photochromic Fuzzy Logic (BIPFUL) systems}

As explained in the introductory preamble, the final step of the analysis of the deterministic part of human colour perception requires the implementation of equation (8). If equation (8) describes properly the basic way humans distinguish colours ${ }^{2}$, by implementing it, we expect to achieve a mechanism suitable to discriminate electromagnetic frequencies. Since humans distinguish visible but not UV frequencies, because the cornea and the lens are not transparent to UV and they prevent UV photons to reach the retina, we have devised sets of appropriately selected thermally reversible direct photochromic compounds to extend human vision to the UV. Direct photochromic species usually absorb just in the UV; upon UV irradiation, they change molecular structure and produce an absorption band into the visible, which confers colour to the system. Such transformation is reversible: after discontinuing the UV irradiation, the colour bleaches. The bleaching rate depends on the compound and the properties of the medium surrounding the photochromic species $[13,14]$.

The photochromic compounds we use in this work for implementing Biologically Inspired Photochromic Fuzzy Logic (BIPFUL) systems that extend human ability to perceive electromagnetic radiation to the UV, are shown in Figs 1 and 3. They are one spirooxazine (1) and four naphthopyrans $(\mathbf{2}, \mathbf{3}, \mathbf{4}, \mathbf{5})$. The absorption spectra of their uncoloured closed forms (Un) are depicted on the left-hand panels of the graphs in Fig. 3, below their molecular structures, whereas

\footnotetext{
${ }^{2}$ In our description, we have not considered the concept of receptive fields wherein the connections between the photosensitive cells and the first two layers of neurons (bipolar and ganglion cells) on the retina play a crucial role.
} 
the absorption bands they produce into the visible under UV irradiation are shown in the right-hand panels, below the pictures showing the colours they originate within a cuvette. Compound (1), whose absorption shows a tail up to the visible, gives rise to a band centred at $611 \mathrm{~nm}$ and its solution becomes blue. Naphtho[1,2-b]pyran (2) that has the smallest values of absorption coefficients in the $387-294 \mathrm{~nm}$ range, gives rise to a peak at $492 \mathrm{~nm}$ with a less intense shoulder at ca. $410 \mathrm{~nm}$ and its solution becomes pink. Naphtho[2,1-b]pyran (3) affords a band with a maximum at $462 \mathrm{~nm}$ and its solution becomes orange. Compound (4) that has an absorption spectrum quite similar to that of (3), produces a band peaked at $413 \mathrm{~nm}$, conferring yellow colour to its solution. Finally, naphthopyran (5) that commences absorption at the shortest wavelengths among the others (below $370 \mathrm{~nm}$ ), originates a band centred at $555 \mathrm{~nm}$ and its solution appears purple.

The criteria we followed to mix the photochromic compounds and generate BIPFUL systems are the following. First, the absorption bands of the closed uncoloured (Un) forms were assumed to be input fuzzy sets. Second, the absorption bands of the open coloured (Col) forms were assumed to be output fuzzy sets. Third, the algorithm expressing the degree of membership of the UV radiation, having intensity $\mathrm{I}_{0}\left(\lambda_{\text {irr }}\right)$ at the wavelength $\lambda_{\text {irr }}$, to the absorption band of the $i$-th compound, in analogy with equation (8), is given by:

$$
\mu_{U V, i}=\Phi_{P C, i}\left(\lambda_{\text {irr }}\right) I_{0}\left(\lambda_{\text {irr }}\right)\left(1-10^{-\varepsilon_{U n, i} C_{0, i} l}\right)
$$

In equation (9), $\Phi_{P C, i}\left(\lambda_{i r r}\right)$ is the photochemical quantum yield, $\varepsilon_{U n, i}$ the absorption coefficient at $\lambda_{\text {irr }}$ for the $i$-th photochromic species, and $C_{0, i}$ is its analytical concentration. Finally, the equation expressing the activation of the $i$-th output fuzzy sets is:

$A_{C o, i}=\frac{\varepsilon_{C o, i}\left(\lambda_{a n}\right)}{k_{\Delta, i}} \mu_{U V, i}$

In equation (10), $A_{C o, i}$ is the absorbance at the wavelength $\lambda_{a n}$ into the visible and due to the coloured form of the $i$-th photochromic species; $\varepsilon_{C o, i}\left(\lambda_{a n}\right)$ is its absorption coefficient, and $k_{\Delta, i}$ is 
its kinetic constant of the bleaching reaction. Finally, $\mu_{U V, i}$ has been defined in equation (8). Each absorption spectrum recorded at the photo-stationary state will be the sum of as many terms represented by equation (10) as there are photochromic components present within the BIPFUL system. Of course, we need to know the photocolouration quantum yields as functions of the irradiation wavelengths and the lifetimes of the coloured forms, to apply equation (10). These data are reported in Table 1 of our previous study [7].

In this work, we have prepared many BIPFUL systems having from three to five photochromic compounds, chosen by applying equations (9) and (10), and we compare their performance in discriminating between the three UV regions: UV-A, UV-B and UV-C. To make a quantitative comparison, we have transformed the spectra, recorded at the photo-stationary states, in vectors of the CIE L*a*b* space and we have calculated the Euclidean distance between pairs of vectors. Moreover, we have calculated their Colourability as defined in equation (7).
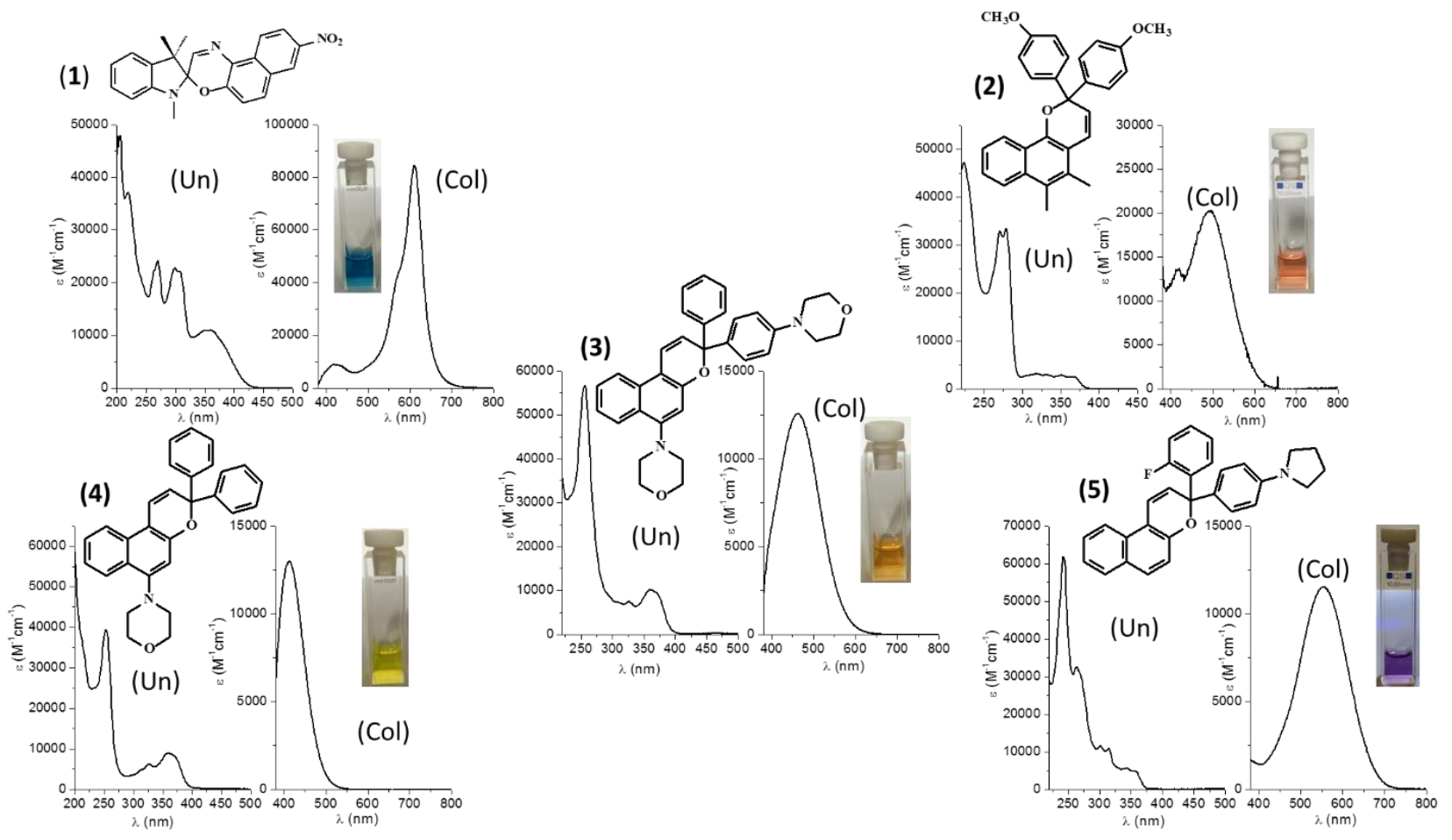
Fig. 3 Structures of the five photochromic species along with the absorption spectra of their closed and open forms and pictures showing the colours of the solutions in cuvettes after UV irradiation.

\subsection{BIPFUL systems with three photochromic compounds}

The ternary BIPFUL systems we have prepared and investigated are listed in Table 1. Compound (1) is always present because it has the most red-shifted spectra for both the uncoloured and the coloured form. Since the maximum value of the absorption coefficient for the coloured form of (1) is about four times larger than those of the other coloured forms derived from (2) - (5), it is convenient that the concentration of (1) is never in excess. In designing ternary BIPFUL systems, it is useful to include either (3) or (4), because they have quite similar absorption spectra in their uncoloured forms. Finally, compounds (2) and (5) have the common feature of absorbing a limited amount of the UV-A frequencies.

\section{Table 1}

Compositions of the ternary BIPFUL systems. The concentrations are expressed in molarity (M).

\begin{tabular}{|c|c|c|c|c|c|}
\hline System & $\mathbf{1}(\mathbf{M})$ & $\mathbf{2}(\mathbf{M})$ & $\mathbf{3}(\mathbf{M})$ & $\mathbf{4}(\mathbf{M})$ & $\mathbf{5}(\mathbf{M})$ \\
\hline $\boldsymbol{A}$ & $4.9 \times 10^{-5}$ & $5.3 \times 10^{-5}$ & $5.3 \times 10^{-5}$ & & \\
\hline $\boldsymbol{B}$ & $1.0 \times 10^{-4}$ & & & $1.0 \times 10^{-4}$ & $1.0 \times 10^{-4}$ \\
\hline $\boldsymbol{C}$ & $5.2 \times 10^{-5}$ & & & $1.5 \times 10^{-4}$ & $3.0 \times 10^{-4}$ \\
\hline $\boldsymbol{D}$ & $5.2 \times 10^{-5}$ & $3.0 \times 10^{-4}$ & & $1.5 \times 10^{-4}$ & \\
\hline $\boldsymbol{E}$ & $1.0 \times 10^{-4}$ & $2.0 \times 10^{-4}$ & & $2.0 \times 10^{-4}$ & \\
\hline $\boldsymbol{F}$ & $5.2 \times 10^{-5}$ & & $1.5 \times 10^{-5}$ & & $3.0 \times 10^{-4}$ \\
\hline
\end{tabular}

The spectra recorded at the photo-stationary states for the six ternary BIPFUL systems are shown in

Fig. 4. The black, red and green traces are the spectra recorded under UV-A, UV-B, and UV-C, respectively. The spectral profiles of the radiation sources used to generate the coloured forms are 
shown in Fig. 2. It is clear that compound (1) contributes appreciably to the shape of the spectra recorded under UV-A and UV-B, but not under UV-C, because the absorption coefficients of (1) in UV-C are smaller than those of the other compounds (see Fig. 3). Moreover, when compound (4) is present (that is, in $\boldsymbol{B}, \boldsymbol{C}, \boldsymbol{D}, \boldsymbol{E}$ BIPFUL systems), it gives rise to an intense shoulder in the blue only under UV-A.
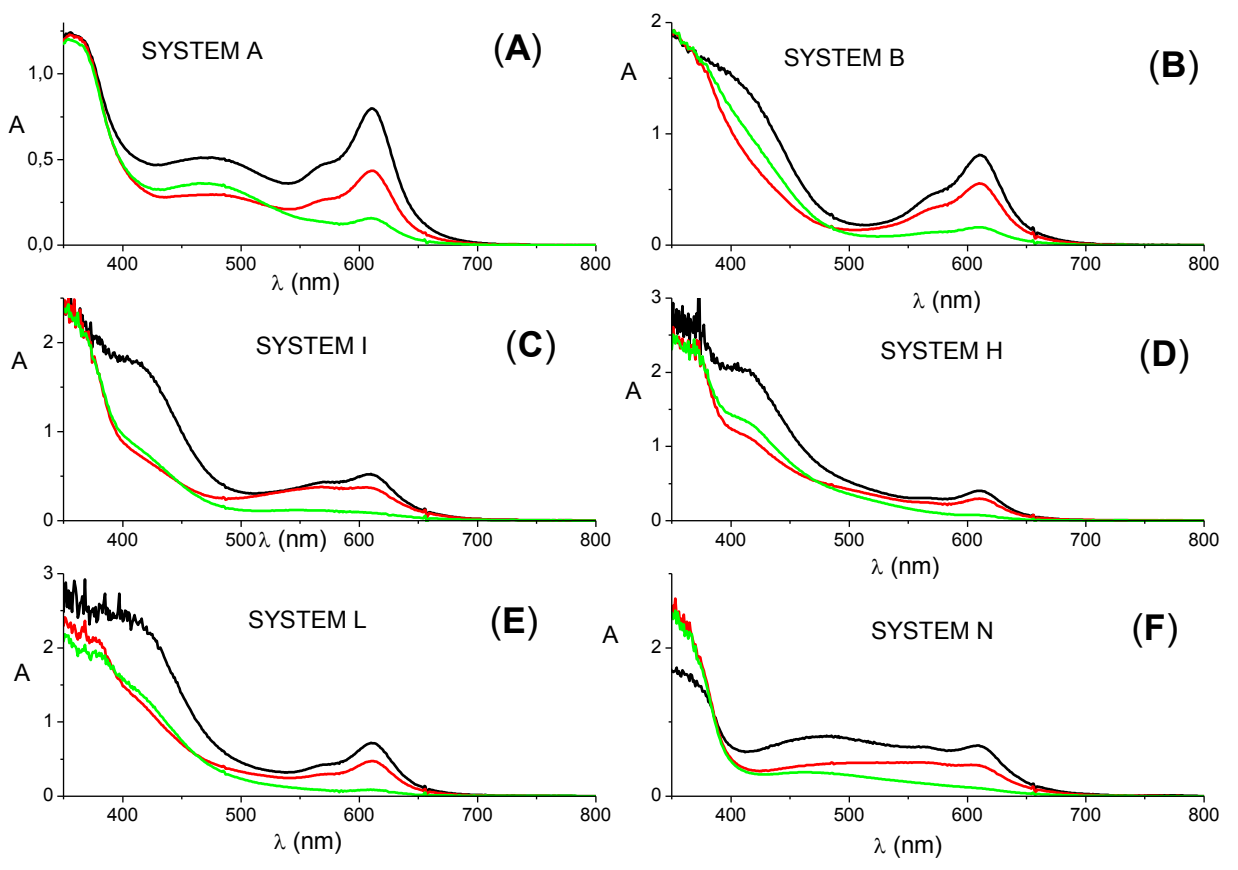

Fig. 4 Spectra recorded at the photo-stationary states upon UV-A (black traces), UV-B (red traces) and UV-C (green traces) for the ternary BIPFUL systems $\boldsymbol{A}, \boldsymbol{B}, \boldsymbol{C}, \boldsymbol{D}, \boldsymbol{E}$ and $\boldsymbol{F}$. The spectral profiles of the radiation sources are depicted in Fig. 2.

All the spectra of Fig. 4 have been transformed in tristimulus values $(\mathrm{X}, \mathrm{Y}, \mathrm{Z}), \mathrm{CIE} \mathrm{L}^{*} \mathrm{a}^{*} \mathrm{~b}^{*}$ and chromaticity coordinates $(\mathrm{x}, \mathrm{y}, \mathrm{z})$, according to the procedures explained in paragraph 2.2 . The results are reported in Table S1 where additionally the colours of the solutions are shown as small squared patches (sections of a photographic image). In Table S1, there is also the value of the 
Chromaticity as defined in equation (7). It is evident that all the ternary BIPFUL systems are suitable for distinguishing between the three regions of the UV, because they give rise to three different hues under UV-A, UV-B and UV-C. However, we want to find the best BIPFUL system for discriminating the three UV regions. For this purpose, we measured the Euclidean distance between pairs of colour vectors plotted in the CIE L*a*b* colour space system and calculated the sum of the values of Colourability achieved for each BIPFUL system. The results are reported in Table 2.

\section{Table 2}

Eucledian distances $D_{i, j}$ (where $i$ can be UV-A or UV-B, whereas $j$ can be UV-B or UV-C) between pairs of vectors in the CIE $\mathrm{L}^{*} \mathrm{a}^{*} \mathrm{~b}^{*}$ for the ternary BIPFUL systems. $\mathrm{C}_{\mathrm{TOT}}$ is the sum of the Colourability values achieved for the same BIPFUL system under UV-A, UV-B and UV-C.

\begin{tabular}{|c|l|l|l|c|}
\hline System & $D_{U V A, U V B}$ & $D_{U V A, U v C}$ & $D_{U V B, U v C}$ & $C_{\text {TOT }}$ \\
\hline $\boldsymbol{A}$ & 13.20 & 24.68 & 21.14 & 0.0147 \\
\hline $\boldsymbol{B}$ & 22.22 & 31.10 & 31.06 & 0.0949 \\
\hline $\boldsymbol{C}$ & 34.25 & 24.09 & 27.79 & 0.1197 \\
\hline $\boldsymbol{D}$ & 20.73 & 15.56 & 20.45 & 0.4319 \\
\hline $\boldsymbol{E}$ & 22.05 & 27.07 & 27.56 & 0.3766 \\
\hline $\boldsymbol{F}$ & 17.21 & 31.59 & 25.02 & 0.0122 \\
\hline
\end{tabular}

As far as the Euclidean distances are concerned, the largest values have been obtained with $\boldsymbol{B}$ and $\boldsymbol{C}$ BIPFUL systems. Both consist of compounds (1), (4) and (5). The reason why $\boldsymbol{B}$ and $\boldsymbol{C}$ BIPFUL systems are the best resides on their excellent spectral match in both the UV and the visible region, as it can be clearly noticed in Fig. 3. However, $\boldsymbol{B}$ and $\boldsymbol{C}$ BIPFUL systems do not have large values of the total Colourability $\left(\mathrm{C}_{\mathrm{TOT}}\right)$ because under UV-B they give rise to neutral grey hues and hence 
to small $\mathrm{C}$ values (cf. paragraph 2.2). As far as $\mathrm{C}_{\mathrm{TOT}}$ is concerned, the best performances have been achieved with $\boldsymbol{D}$ and $\boldsymbol{E}$ BIPFUL systems. Both of them contain compounds (1), (2) and (4). The presence of (2) instead of (5) guarantees more colour at the photo-stationary states. In fact, although the photo-colouration quantum yields for $(2)\left(\Phi_{\mathrm{PC}}(\mathrm{UV}-\mathrm{A})=0.17, \Phi_{\mathrm{PC}}(\mathrm{UV}-\mathrm{B})=0.26, \Phi_{\mathrm{PC}}(\mathrm{UV}-\mathrm{C})=\right.$ $0.45)$ [7] are quite similar to those for $(5)\left(\Phi_{\mathrm{PC}}(\mathrm{UV}-\mathrm{A})=0.28, \Phi_{\mathrm{PC}}(\mathrm{UV}-\mathrm{B})=0.23, \Phi_{\mathrm{PC}}(\mathrm{UV}-\mathrm{C})=\right.$ 0.62) [7], the lifetime of the (2) coloured form $\left(\tau=\left(\frac{1}{k_{\Delta}}\right)=37 s\right)$ [7] is almost twice larger than that of $(5)\left(\tau=\left(\frac{1}{k_{\Delta}}\right)=19 s\right)[7]$.

\subsection{BIPFUL systems with four or five photochromic compounds}

We have also studied the performance of a selection of BIPFUL systems with four and five photochromic species. The list is reported in Table 3. Compounds (1) and (5) are always present because they are the compounds that absorb the widest and shortest portions of UV, respectively (see Figure 3).

\section{Table 3}

Compositions of BIPFUL systems with four and five photochromic compounds. The concentrations are expressed in molarity (M).

\begin{tabular}{|c|c|c|c|c|c|}
\hline System & $\mathbf{1}(\mathbf{M})$ & $\mathbf{2}(\mathbf{M})$ & $\mathbf{3}(\mathbf{M})$ & $\mathbf{4}(\mathbf{M})$ & $\mathbf{5}(\mathbf{M})$ \\
\hline $\boldsymbol{G}$ & $3.7 \times 10^{-5}$ & $4.0 \times 10^{-5}$ & & $4.0 \times 10^{-5}$ & $4.2 \times 10^{-5}$ \\
\hline $\boldsymbol{H}$ & $2.0 \times 10^{-5}$ & $5.4 \times 10^{-5}$ & & $3.2 \times 10^{-5}$ & $5.5 \times 10^{-5}$ \\
\hline $\boldsymbol{I}$ & $3.7 \times 10^{-5}$ & $4.0 \times 10^{-5}$ & $4.0 \times 10^{-5}$ & & $4.2 \times 10^{-5}$ \\
\hline $\boldsymbol{L}$ & $3.7 \times 10^{-5}$ & & $4.0 \times 10^{-5}$ & $4.0 \times 10^{-5}$ & $4.2 \times 10^{-5}$ \\
\hline $\boldsymbol{M}$ & $4.3 \times 10^{-5}$ & $1.7 \times 10^{-4}$ & & $1.2 \times 10^{-4}$ & $1.7 \times 10^{-4}$ \\
\hline $\boldsymbol{N}$ & $4.3 \times 10^{-5}$ & $1.7 \times 10^{-4}$ & $1.2 \times 10^{-4}$ & & $1.7 \times 10^{-4}$ \\
\hline $\boldsymbol{O}$ & $5.2 \times 10^{-5}$ & $1.4 \times 10^{-4}$ & & $7.4 \times 10^{-5}$ & $1.4 \times 10^{-4}$ \\
\hline $\boldsymbol{P}$ & $2.9 \times 10^{-5}$ & $3.2 \times 10^{-5}$ & $3.2 \times 10^{-5}$ & $3.2 \times 10^{-5}$ & $3.3 \times 10^{-5}$ \\
\hline $\boldsymbol{Q}$ & $3.5 \times 10^{-5}$ & $1.4 \times 10^{-4}$ & $1.0 \times 10^{-4}$ & $9.8 \times 10^{-5}$ & $1.3 \times 10^{-4}$ \\
\hline
\end{tabular}


The spectra recorded at the photostationary states for the seven BIPFUL systems with four photochromes $(\boldsymbol{G}, \boldsymbol{H}, \boldsymbol{I}, \boldsymbol{L}, \boldsymbol{M}, \boldsymbol{N}, \boldsymbol{O})$ and for the two, $\boldsymbol{P}$ and $\boldsymbol{Q}$, having five components, are depicted in Fig. 5. The black, red and green traces are the spectra recorded under UV-A, UV-B, and UV-C, respectively. All those spectra can be reproduced by using equation (10) (see reference [7]), knowing that the profiles of the radiation sources are those shown in Fig. 2.

The open form of compound (1) contributes appreciably to the shape of the spectra recorded under UV-A and UV-B, but not under UV-C as it occurs in the case of ternary BIPFUL systems. Whenever we have compound (4) (in all cases except $\boldsymbol{I}$ and $\boldsymbol{N}$ ), it always gives rise to a shoulder into the blue under UV-A, sometimes also under UV-C, but never under UV-B. This occurs because the absorption coefficient of (4) in UV-B is quite small.
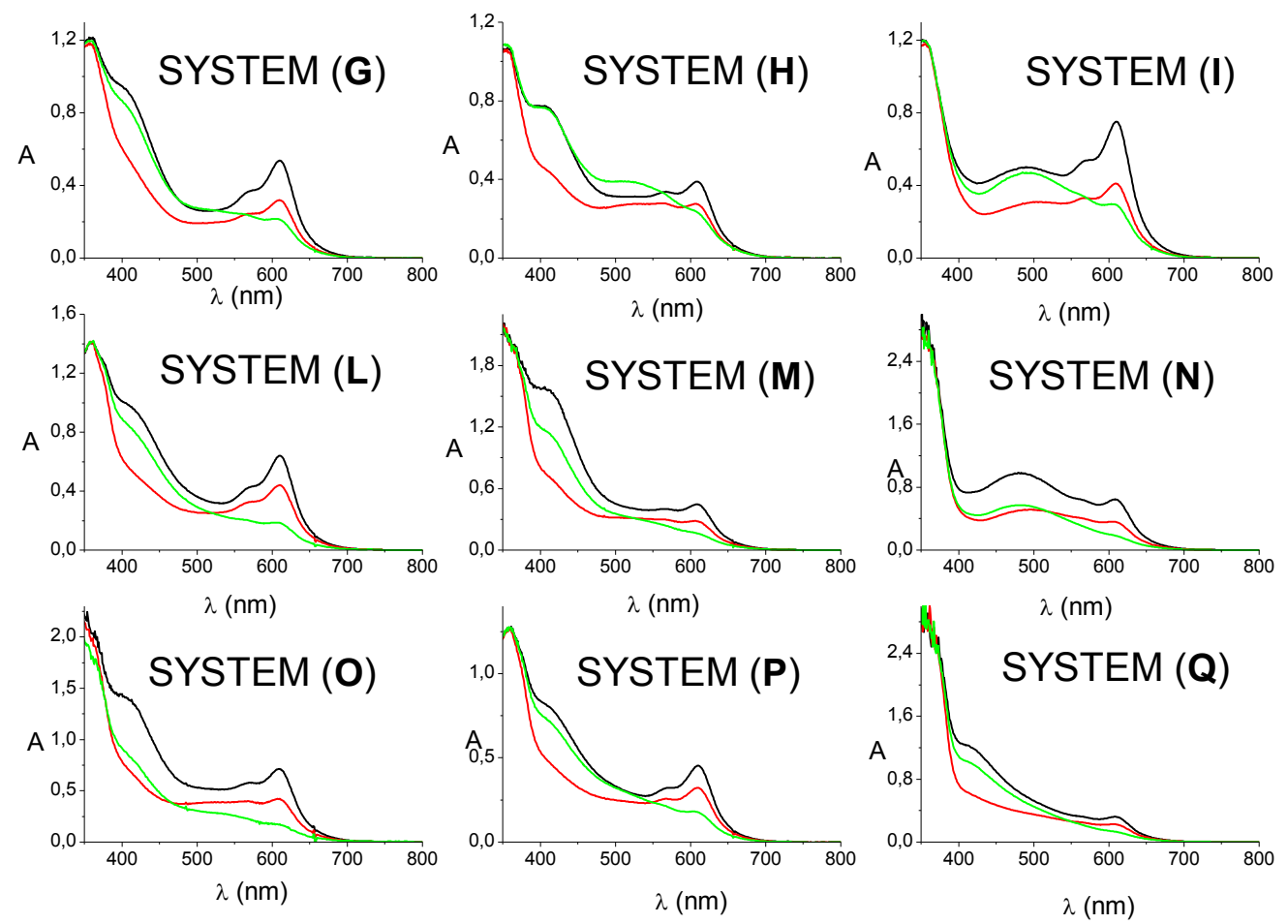

Fig. 5 Spectra recorded at the photo-stationary states upon UV-A (black traces), UV-B (red traces) and UV-C (green traces) for the four-photochromes BIPFUL systems $\boldsymbol{G}, \boldsymbol{H}, \boldsymbol{I}, \boldsymbol{L}, \boldsymbol{M}, \boldsymbol{N}, \boldsymbol{O}$, and for the five-photochromes BIPFUL systems $\boldsymbol{P}$ and $\boldsymbol{Q}$. 
All the spectra of Fig. 5 have been transformed in tristimulus values (X, Y, Z), CIE L*a*b* and chromaticity coordinates $(\mathrm{x}, \mathrm{y}, \mathrm{z})$, according to the procedures explained in paragraph 2.2 . The results are reported in Table S2 where the colours of the solutions are shown as small squared patches (sections of a photographic image). In Table S2, there is also the value of the Chromaticity as defined in equation (7). It is apparent that even the BIPFUL systems with four and five photochromes are effective in discriminating the three UV regions. They create colours that have distinct hues. However, it is interesting to compare quantitatively their performances. For this purpose, we exploit the calculation of the Eucledian distances between pairs of vectors in the CIE $\mathrm{L}^{*} \mathrm{a}{ }^{*} \mathrm{~b}^{*}$ space and the values of overall Colourability $\left(\mathrm{C}_{\mathrm{TOT}}\right)$ for each BIPFUL system (see Table 4).

\section{Table 4}

Eucledian distances $D_{i, j}$ between pairs of vectors in the CIE L*a* $\mathrm{b}^{*}$ space representing the colours generated by irradiation with UV-A, UV-B and UV-C. $\mathrm{C}_{\mathrm{TOT}}$ is the sum of the Colourability achieved for the same BIPFUL system under UV-A, UV-B and UV-C.

\begin{tabular}{|c|l|l|l|c|}
\hline System $\left(\boldsymbol{\lambda}_{\text {irr }}\right)$ & $\boldsymbol{D}_{\text {UVA,UVB }}$ & $\boldsymbol{D}_{\text {UVA,UVC }}$ & $\boldsymbol{D}_{\text {UVB,UVC }}$ & $\mathbf{C}_{\text {TOT }}$ \\
\hline $\boldsymbol{G}$ & 14.64 & 19.66 & 18.18 & 0.03487 \\
\hline $\boldsymbol{H}$ & 14.47 & 14.66 & 17.39 & 0.0275 \\
\hline $\boldsymbol{I}$ & 12.67 & 21.17 & 17.40 & 0.0127 \\
\hline $\boldsymbol{L}$ & 18.95 & 22.93 & 27.84 & 0.07321 \\
\hline $\boldsymbol{M}$ & 26.96 & 13.85 & 24.30 & 0.1900 \\
\hline $\boldsymbol{N}$ & 20.78 & 20.47 & 17.51 & 0.0539 \\
\hline $\boldsymbol{O}$ & 21.72 & 23.23 & 22.20 & 0.0650 \\
\hline $\boldsymbol{P}$ & 16.00 & 24.69 & 20.95 & 0.0560 \\
\hline
\end{tabular}




\begin{tabular}{|l|l|l|l|l|}
\hline$Q$ & 24.17 & 9.51 & 25.66 & 0.2563 \\
\hline
\end{tabular}

If we consider the sum of the Eucledian distances for each BIPFUL system, we find that the best performances have been obtained with $\boldsymbol{L}\left(\sum D_{i, j}=69.72\right), \boldsymbol{M}\left(\sum D_{i, j}=65.11\right)$ and $\boldsymbol{O}\left(\sum D_{i, j}=\right.$ 67.15). Both $\boldsymbol{M}$ and $\boldsymbol{O}$ contain compounds (1), (2), (4) and (5). As far as the total Colourability is concerned, the largest values have been achieved with the $\boldsymbol{Q}$ and $\boldsymbol{M}$ BIPFUL systems. However, $\boldsymbol{Q}$ has a quite small $\boldsymbol{D}_{\text {uva,uvc }}$ (see also Table S2). The systems $\boldsymbol{L}$ and $\boldsymbol{O}$ give small $\mathrm{C}_{\mathrm{TOT}}$ because under UV-B they generate a colour having a grey hue (see Table S2).

\section{Conclusions}

All of the BIPFUL systems, which have been studied, are able to discriminate between the three regions of the UV spectrum: UV-A, UV-B and UV-C. The operationally simple ternary BIPFUL systems generally show better performance than the BIPFUL systems with either the four or five components which have been studied. However, there is a caveat to the foregoing statement, in that the response of a multi-component BIPFUL system is relative to the spectral, photochemical and dynamical properties of the pre-selected photochromic compounds; if a broader range of initial photochromes were available for selection, then a much improved four or five component BIPFUL system may result. Among all the BIPFUL systems studied, the $\boldsymbol{C}$ system guaranteed the largest Euclidean distances between the CIEL*a*b* vectors, but not the largest total Colourability $\left(\mathrm{C}_{\mathrm{TOT}}\right)$ because under UV-B it produced a grey colour. In terms of $\mathrm{C}_{\mathrm{TOT}}$, the best result has been achieved with the ternary $\boldsymbol{D}$ BIPFUL system.

This study provides valuable indications for designing even better BIPFUL systems to discriminate between the UV frequencies. In fact, further improvements can be obtained by 
synthesizing other photochromic compounds, having more distinct spectral profiles in the UV, and giving rise to more distinct and less overlapping bands into the visible region. The criteria used to define the composition of our BIPFUL systems can work as guiding principles for the design of the new photochromic compounds.

Finally, this work demonstrates that a system of properly selected chemical compounds can exhibit a computational power higher than that of their single constituents. In fact, every photochromic compound is an "optical transistor" [15-17] that can be suitable to implement even complicated binary and fuzzy logic gates [18]. However, the Biologically Inspired Photochromic Fuzzy Logic systems, described in this paper, show the emergent property of discriminating the three UV regions of the electromagnetic spectrum. They mimic the computational power of vertebrates and invertebrates that have distinct photoreceptors to distinguish between frequencies of the electromagnetic spectrum. This study proves that the development of Systems Chemistry can be really fruitful for the development of Chemical Artificial Intelligence.

\section{Acknowledgments}

P. L. Gentili acknowledges the financial support by the University of Perugia (Fondo Ricerca di Base 2014, D. D. n. 170, 23/12/2014). A. L. Rightler thanks the American Chemical Society IREU Program and the National Science Foundation for support under award number DMR-1262908.

\section{References}

[1] Goldreich O. Computational Complexity. A Conceptual Perspective. Cambridge University Press, New York; 2008.

[2] Rozenberg G, Bäck T, Kok JN. Handbook of Natural Computing, Berlin: Springer, 2012. 
[3] Gentili PL. Small steps towards the development of chemical artificial intelligent systems. RSC Adv 2013; 3: 25523-25549.

[4] Gallistel CR, King A. Memory and the computational brain: Why cognitive science will transform neuroscience. New York: Blackwell/Wiley; 2009.

[5] Marr D. Vision. A computational investigation into the human representation and processing of visual information. The MIT Press; 2010.

[6] Ludlow RF, Otto S. Systems Chemistry. Chem. Soc. Rev. 2008; 37, 101-108.

[7] Gentili PL, Rightler AL, Heron BM, Gabbutt CD. Extending human perception of electromagnetic radiation to the UV region through biologically inspired photochromic fuzzy logic (BIPFUL) systems. Chem. Commun. 2016; 52, 1474-1477.

[8] Gentili PL. The Fuzziness of a Chromogenic Spirooxazine. Dyes and Pigments 2014; 110: 235248.

[9] Paxinos G, Mai JK. The Human Nervous System, $2^{\text {nd }}$ edn, Academic Press, 2004.

[10] Gentili PL. The human sensory system as a collection of specialized Fuzzifiers: a conceptual framework to inspire new artificial intelligent systems computing with words. J Intel Fuzzy Sys. 2014; 27: 2137-2151.

[11] Zadeh LA. Fuzzy sets, Information and Control 1965; 8(3):338-353.

[12] Oyster CW. The Human Eye: Structure and Function. Sinauer Associates, Inc., Sunderland, MA, 1999.

[13] Dürr H, Bouas-Laurent H. Photochromism: Molecules and Systems. Elsevier, Revised Edition, Amsterdam, The Netherlands, 2003. 
[14] Zhang J, Zou Q, Tian H. Photochromic Materials: More Than Meets The Eye. Adv. Mater. 2013; 25: 378-399.

[15] Gust D, Andréasson J, Pischel U, Moore TA, Moore AL. Data and signal processing using photochromic molecules. Chem. Commun. 2012; 48: 1947 - 1957.

[16] Szaciłowski K. Digital Information Processing in Molecular Systems. Chem. Rev. 2008; 108: 3481-3548.

[17] Wu Y, Xie Y, Zhang Q, Tian H, Zhu W, Li A. D. Q. Quantitative Photoswitching in Bis(dithiazole)ethene Enables Modulation of Light for Encoding Optical Signals. Angew. Chem. Int. Ed. 2014; 53: 2090 -2094.

[18] Gentili PL. The fundamental Fuzzy logic operators and some complex Boolean logic circuits implemented by the chromogenism of a spirooxazine. Phys. Chem. Chem. Phys. 2011; 13: 2033520344.

\section{Graphical Abstract}

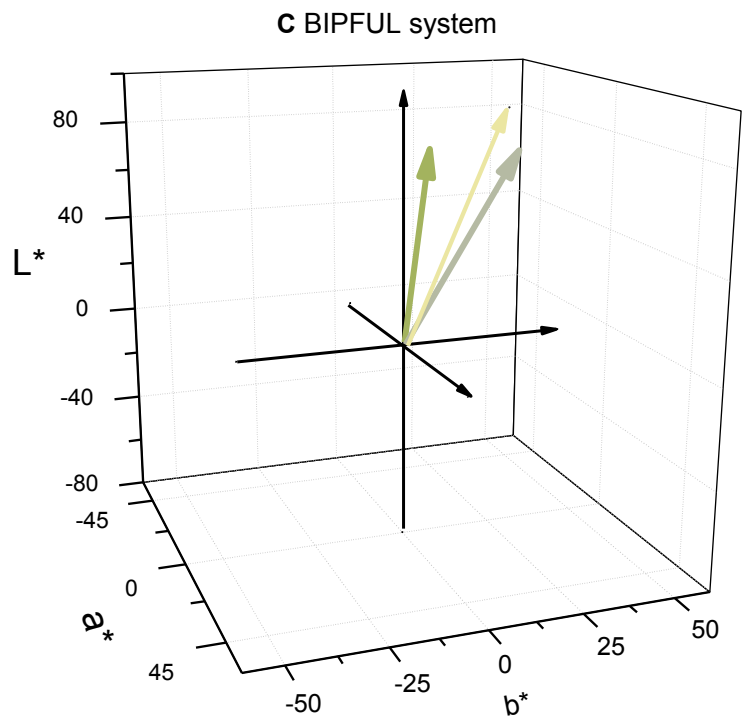

UVA, UVB and UVC radiation is distinguished through the selective activation of photochromic systems and analysis of their response using Fuzzy Logic and CIE colour space. 